\title{
Detection of Targetable Genetic Alterations in Korean Lung Cancer Patients: A Comparison Study of Single-Gene Assays and Targeted Next-Generation Sequencing
}

\section{Eunhyang Park, MD, PhD \\ Hyo Sup Shim, MD, PhD}

Department of Pathology, Severance Hospital, Yonsei University College of Medicine, Seoul, Korea
Correspondence: Hyo Sup Shim, MD, PhD

Department of Pathology, Severance Hospital,

Yonsei University College of Medicine,

50 Yonsei-ro, Seodaemun-gu, Seoul 03722, Korea

Tel: 82-2-2228-1760

Fax: 82-2-362-0860

E-mail: shimhs@yuhs.ac

Received June 3, 2019

Accepted November 8, 2019

Published Online November 8, 2019

\begin{abstract}
Purpose
Epidermal growth factor receptor (EGFR), anaplastic lymphoma kinase (ALK), and ROS proto-oncogene 1 (ROS1) are 'must-test' biomarkers in the molecular diagnostics of advanced-stage lung cancer patients. Although single-gene assays are currently considered the gold standard for these genes, next-generation sequencing (NGS) tests are being introduced to clinical practices. We compared the results of current diagnostics and aimed to suggest timely effective guidance for their clinical use.
\end{abstract}

\section{Materials and Methods}

Patients with lung cancer who received both conventional single-gene assays and subsequent targeted NGS testing were enrolled, and the results of their tests were compared.

\section{Results}

A total of 241 patients were enrolled, and the EGFR real-time polymerase chain reaction, ALK fluorescence in situ hybridization (FISH), and ROS1 FISH assays exhibited $92.9 \%$, $99.6 \%$, and $99.5 \%$ concordance with the NGS tests, respectively. The discordant cases were mostly false-negatives of the single-gene assays, probably due to technical limitation. Of 158 cases previously designated as wild-type, EGFR, ALK, and ROS1 alterations were identified in $10.1 \%, 1.9 \%$, and $1.3 \%$, respectively, and other targetable alterations were identified in $36.1 \%$ of the cases. Of patients with additionally identified actionable alterations, $32.6 \%$ (31/95) received matched therapy with a clinical benefit of $48.4 \%$ (15/31).

\section{Conclusion}

Even though the conventional and NGS methods were concordant in the majority of cases, NGS testing still revealed a considerable number of additional EGFR, ALK, and ROS1 alterations, as well as other targetable alterations, in Korean advanced-stage lung cancer patients. Given the high frequency of EGFR and other targetable mutations identified in the present study, NGS testing is highly recommended in the diagnosis of Korean lung cancer patients.

\section{Introduction}

The discovery of oncogenic alterations with sensitivity to tyrosine kinase inhibitors (TKIs) has changed the therapeutic landscape of lung cancer. In particular, epidermal growth factor receptor (EGFR) mutations, anaplastic lymphoma

\section{Key words}

Lung neoplasms, Molecular diagnostics,

Next-generation sequencing, Epidermal growth factor receptor, Anaplastic lymphoma kinase, ROS proto-oncogene 1 (ROS1) 
tests, are considered the gold standard for selecting eligible patients for EGFR-, ALK-, and ROS1-specific TKI therapy $[1,2]$. However, with the development of next-generation sequencing (NGS), other less common oncogenic alterations with available therapies have been detected [3].

NGS is a high-throughput multiplex sequencing method which is capable of identifying a variety of genetic alterations simultaneously. In addition, targeted NGS carries out deep sequencing of relevant targets and detects mutations with low mutation levels with high sensitivity. Because of these advantages, many studies have investigated the feasibility of using NGS methods in clinical practice [4-6]. In Korea, as National Health Insurance reimbursement became available in 2017, NGS testing has increasingly been used as a clinical diagnostic method. However, high costs, specialized implements and bioinformatics, complex test processes, and relatively long turnaround time hinder its implementation as a standard method for detecting genetic alterations [1].

In this study, we aimed to compare the results of singlegene assays and NGS testing for EGFR, ALK, and ROS1 and assess the occurrence of false results associated with these methods in the molecular diagnostics of lung cancer patients. In addition, a comprehensive algorithm for selecting patients for TKIs is proposed, which is not to leave appropriately treatable patients behind.

\section{Materials and Methods}

\section{Patients}

Lung cancer patients who received NGS testing at Yonsei University Severance Hospital (Seoul, Korea) between July 2017 and March 2019 were enrolled. Clinical data, including age, sex, and smoking history, were obtained from the patients' medical records.

\section{Single-gene EGFR assay}

To detect EGFR mutations, peptide nucleic acid (PNA)mediated real-time PCR-based methods were performed using the PNAClamp EGFR Mutation Detection Kit (Panagene, Daejeon, Korea) or PANAMutyper EGFR Kit (Panagene) according to manufacturer's instructions. In PNAClamp method, the efficiency and results of the test is determined by measuring threshold cycle $(\mathrm{Ct})$ value. $\mathrm{Ct}$ value is a PCR cycle number at which the fluorescent signal of the reaction crosses the threshold and it is inversely related to the starting amount of target DNA. For data interpretation, PNA clamped Ct value and non-PNA Ct value of patient samples are measured. If non-PNA Ct value is between 22 and 30, the sample is regarded to have an appropriate quality. In addition, delta $\mathrm{Ct}(\Delta \mathrm{Ct})$ values $(\Delta \mathrm{Ct} 1=$ standard $\mathrm{Ct}-$ sample PNA Ct, $\Delta \mathrm{Ct} 2=$ sample PNA Ct-sample non-PNA Ct) are calculated. $\Delta \mathrm{Ct} 1<0$ indicates target mutation wild-type of tested samples, while (1) $\Delta \mathrm{Ct} 1 \geq 2$, or $(2) 0<\Delta \mathrm{Ct} 1<2$ and $\Delta \mathrm{Ct} 2 \leq 3$ is regarded presence of targeted mutation. The manufacturer also described a possibility of suboptimal tests, if $\Delta \mathrm{Ct} 1$ is between 0 and 2 and non-PNA $\mathrm{Ct}$ value is between 24 and 30. In this case, the sample might have a low mutation rate that re-test by using twice as high concentration of the sample is recommended.

\section{Single-gene $A L K$ and $R O S 1$ assays}

To identify ALK and ROS1 rearrangements, IHC was performed using ALK (rabbit monoclonal, clone D5F3, Cell Signaling Technology, Danvers, MA) and ROS1 (rabbit monoclonal, clone D4D6, Cell Signaling Technology) antibodies, as previously described [7]. For IHC positive cases, FISH was performed using a break-apart ALK or ROS1 probe (Vysis LSI Dual Color, Break Apart Rearrangement Probe, Abbott Molecular, Abbot Park, IL), and ALK or ROS1 rearrangements were scored as positive when at least $15 \%$ of the tumor cells exhibited split or isolated 3 ' signals.

\section{NGS analysis}

Targeted DNA and RNA sequencing were performed using TruSight Tumor 170 (Illumina, San Diego, CA) or a customized cancer panel (NgeneBio, Seoul, Korea). The TruSight Tumor 170 panel was designed to detect 170 cancer-related genes, including 151 genes with potential single nucleotide variants (SNVs) and indels, 59 genes with potential amplifications, and 55 genes with fusion and splice variants (S1 Table). The customized cancer panel was designed to detect 46 cancer-related genes, including 46 genes with potential SNVs and indels, 20 genes with potential amplification, and 17 genes with potential fusion variants (S2 Table). Briefly, $40 \mathrm{ng}$ of formalin-fixed paraffin-embedded (FFPE) tissue-derived DNA and RNA were extracted using Qiagen AllPrep DNA/ RNA FFPE Kit (Qiagen, Hilden, Germany). After hybridization capture-based target enrichment, pairedend sequencing $(2 \times 150 \mathrm{bp})$ was performed using a NextSeq sequencer (Illumina) according to the manufacturer's instructions. Variants with a total depth of at least $100 \times$ and variant allele frequency of at least 3\% was included for analysis. Variant interpretation was based on recommendations from the Association for Molecular Pathology, American Society of Clinical Oncology, and College of American Pathologists [8]. Actionable genetic alterations were stratified into one of four levels based on OncoKB website (http:// www.OncoKB. 
org). Tier 1 variant included level 1 and level 2 genetic alterations that are Food and Drug Administration-approved biomarkers and standard of care. Tier 2 variant included alterations with compelling clinical or preclinical evidence to drug response.

\section{Ethical statement}

The present study was approved by the Institutional Review Board of Severance Hospital (No. 4-2019-0273), and the requirement for written informed consent from the patients was waived, owing to the retrospective nature of the study.

\section{Results}

\section{Patients}

A total of 241 patients were enrolled in the present study (Table 1). The patients included 110 men $(45.6 \%)$ and 131 women $(54.4 \%)$, and their age ranged from 28 to 85 years (mean, 60.1 years). In addition, the population included 156 never smokers $(64.7 \%), 64$ former smokers $(26.6 \%)$, and 21 current smokers $(8.7 \%)$. The histological types consisted of 201 adenocarcinomas $(83.4 \%), 11$ non-small cell carcinomasnot otherwise specified (4.6\%), seven squamous cell carcinomas $(2.9 \%)$, six invasive mucinous adenocarcinomas $(2.5 \%)$, six small cell carcinomas $(2.5 \%)$, four sarcomatoid carcinomas $(1.7 \%)$, three adenosquamous carcinomas $(1.2 \%)$, and three carcinoid tumors (1.2\%). In NGS analysis, 205 cases (85.1\%) were tested with the TruSight Tumor 170 panel and $36(14.9 \%)$ cases were tested with the customized cancer panel. The NGS-tested specimens included 178 biopsies (73.9\%), 62 resections $(25.7 \%)$, and one cytology (0.4\%). The average total read depth of the tests was approximately $1,000 \times$.

All patients had previously received the PCR-based EGFR and ALK IHC/FISH tests at the time of their initial diagnosis. However, in one case, the ALK IHC and FISH tests had failed, due to inadequate sample quality. In addition, 210 patients $(87.1 \%)$ had also received either the ROS1 IHC test, ROS1 FISH test, or both. The remaining 31 patients (12.9\%) had initially been diagnosed at other hospitals and were subject to NGS testing at Severance Hospital without receiving either the ROS1 IHC or ROS1 FISH analyses. All the NGS tests were requested by corresponding oncologists with the aim of identifying therapeutic targets or any alterations of drug resistance in advanced-stage lung cancer patients. The single-gene assays identified EGFR, ALK, and ROS1 alter-
Table 1. Patient characteristics

\begin{tabular}{lc} 
Characteristic & No. $(\%)(\mathbf{n}=\mathbf{2 4 1})$ \\
\hline Age, mean \pm SD (yr) & $60.1 \pm 11.1$ \\
Sex & \\
Male & $110(45.6)$ \\
\hline Female & $131(54.4)$ \\
Smoking & \\
\hline Never smoker & $156(64.7)$ \\
\hline Former smoker & $64(26.6)$ \\
\hline Current smoker & $21(8.7)$ \\
Tumor site & $142(58.9)$ \\
\hline Primary & $99(41.1)$ \\
\hline Metastatic & \\
Diagnosis & $201(83.4)$ \\
\hline ADC & $7(2.9)$ \\
\hline SqCC & $3(1.2)$ \\
\hline Adenosquamous carcinoma & $4(1.7)$ \\
\hline Sarcomatoid carcinoma & $6(2.5)$ \\
\hline IMA & $11(4.6)$ \\
\hline NSCLC-NOS & $6(2.5)$ \\
\hline Small cell carcinoma & $3(1.2)$ \\
\hline Carcinoid tumor & $178(73.9)$ \\
\hline Specimen type & $62(25.7)$ \\
\hline Biopsy & $1(0.4)$ \\
\hline Resection & \\
\hline Fluid cytology & \\
\hline
\end{tabular}

$\mathrm{SD}$, standard deviation; $\mathrm{ADC}$, adenocarcinoma; $\mathrm{SqCC}$, squamous cell carcinoma; IMA, invasive mucinous adenocarcinoma; NSCLC-NOS, non-small cell lung carcinoma (not otherwise specified).

ations in $66(27.4 \%)$, five $(2.1 \%)$, and $13(5.4 \%)$ cases, respectively, whereas NGS testing identified $82(34.0 \%)$, eight $(3.3 \%)$, and $14(5.8 \%)$ cases. The EGFR, ALK, and ROS1 alterations were identified with mutual exclusivity.

\section{Comparison of single-gene and NGS EGFR assays}

Of the 241 cases screened for EGFR mutations, 224 cases (92.9\%) yielded concordant PCR and NGS results, whereas 17 (7.1\%) yielded discordant results (Table 2). Of the 66 EGFR PCR-positive cases, all cases returned positive NGS results, however, one $(1.5 \%$ ) had discordant result. In the discordant case, the PCR method had detected a deletion in exon 19 (E19del), whereas the NGS test had detected p.L747P in exon 19. Meanwhile, for the 175 PCR-confirmed wild-type cases, $159(90.9 \%)$ yielded concordant PCR and NGS results. For the 16 discordant cases (9.1\%), NGS testing revealed new EGFR mutations, including eight hotspot mutations and eight rare mutations. 
Table 2. Summary of single-gene assay and NGS results

\begin{tabular}{|c|c|c|c|c|}
\hline & NGS positive & NGS negative & Total & Concordance rate $(\%)$ \\
\hline \multicolumn{5}{|l|}{ EGFR } \\
\hline PCR positive & $66^{\text {a) }}$ & 0 & 66 & 92.9 \\
\hline PCR negative & 16 & 159 & 175 & \\
\hline Total & 82 & 159 & 241 & \\
\hline \multicolumn{5}{|l|}{$A L K$} \\
\hline FISH positive & 5 & 0 & 5 & 99.2 \\
\hline FISH negative & 2 & 233 & 235 & \\
\hline FISH failure & 1 & 0 & 1 & \\
\hline Total & 8 & 233 & 241 & \\
\hline \multicolumn{5}{|l|}{ ROS1 } \\
\hline FISH positive & 12 & 1 & 13 & 99.5 \\
\hline FISH negative & 0 & 197 & 197 & \\
\hline FISH not done & 2 & 29 & 31 & \\
\hline Total & 14 & 227 & 241 & \\
\hline
\end{tabular}

NGS, next-generation sequencing; EGFR, epidermal growth factor receptor; PCR, polymerase chain reaction; $A L K$, anaplastic lymphoma kinase; FISH, fluorescence in situ hybridization; ROS1, ROS proto-oncogene 1. ${ }^{\text {a) }}$ One of the positive PCR results was a false-positive because a base substitution was interpreted as a deletion mutation.

The eight EGFR hotspot mutations were the L858R mutation (five cases), E19del (p.E746_A750del; two cases), and an insertion in exon 20 (E20ins; p.H773delinsPNPY; one case) which could be detected by PCR methods (Table 3). For five cases, the PCR and NGS testing were performed on the same samples, whereas for the other three cases, different samples were used for PCR and NGS testing. Furthermore, Ct values could be reviewed for four cases. Two cases yielded negative $\Delta \mathrm{Ct} 1$ values and non-PNA Ct values between 22 and 30, which indicated as negative results with appropriate sample quality. In the reports of other two cases, $\Delta \mathrm{Ct} 1$ values between 0 and $2, \Delta C t 2$ values $\geq 3$, and their non-PNA Ct values were between 24 and 30, which are interpreted as negative results with low sample quality.

The other eight PCR-negative NGS-positive cases harbored EGFR mutations that were not targeted by the PCR method. Of these, three cases were exon 18 indel or exon 19 deletion and five cases were E20ins mutations. Four patients (50\%) were treated with EGFR TKI (Table 4). Patient A04 was treated with gefitinib after identifying an EGFR mutation (p.A750_I759>PL) and exhibited a partial response. However, treatment of other patients is ongoing that their therapeutic responses are unknown yet.

\section{Comparison of single-gene and NGS ALK assays}

A total of 238 cases $(99.2 \%)$ yielded concordant ALK FISH and NGS results, and two cases $(0.8 \%)$ yielded discordant results (S3 Table). NGS testing confirmed that all the ALK FISH-positive cases possessed ALK rearrangements. How- ever, NGS testing also identified ALK rearrangements for two of the 235 ALK FISH-negative cases. These two cases were initially diagnosed at other hospitals, which had shown positive ALK IHC results $(+2 / 3)$, but negative ALK FISH results, with $8 \%$ and $9 \%$ of split signals, respectively.

\section{Comparison of single-gene and NGS ROS1 assays}

A total of 209 cases (99.5\%) yielded concordant ROS1 FISH and NGS results, and only one case $(0.5 \%)$ yielded discordant results. For the discordant case, the ROS1 IHC test returned diffuse moderate protein expression $(+2 / 3)$, and the ROS1 FISH test returned $22 \%$ of split signals, whereas NGS identified non-functional (out-of-frame) RNR2 (chromosome 14)ROS1 (chromosome 6) fusion and newly identified the deletion EGFR mutation in exon 18. The result of ROS1 realtime PCR companion diagnostics was also negative.

\section{Genetic alterations in previously documented wild-type cases}

Of the 158 cases denoted as wild-type on the basis of single-gene assays, NGS testing identified 95 patients (60.1\%) who harbored actionable alterations. In addition to 16 EGFR mutations (10.1\%), the NGS testing revealed three ALK fusions (1.9\%), two ROS1 fusions (1.3\%), five BRAF mutations (3.2\%), seven $M E T$ exon 14 skipping or amplification (4.4\%), nine RET fusions (5.7\%), one NTRK2 fusion (0.6\%) and so on (Fig. 1). Of 95 patients with actionable alterations, $32.6 \%(31 / 95)$ received matched therapy and $48.4 \%(15 / 31)$ 


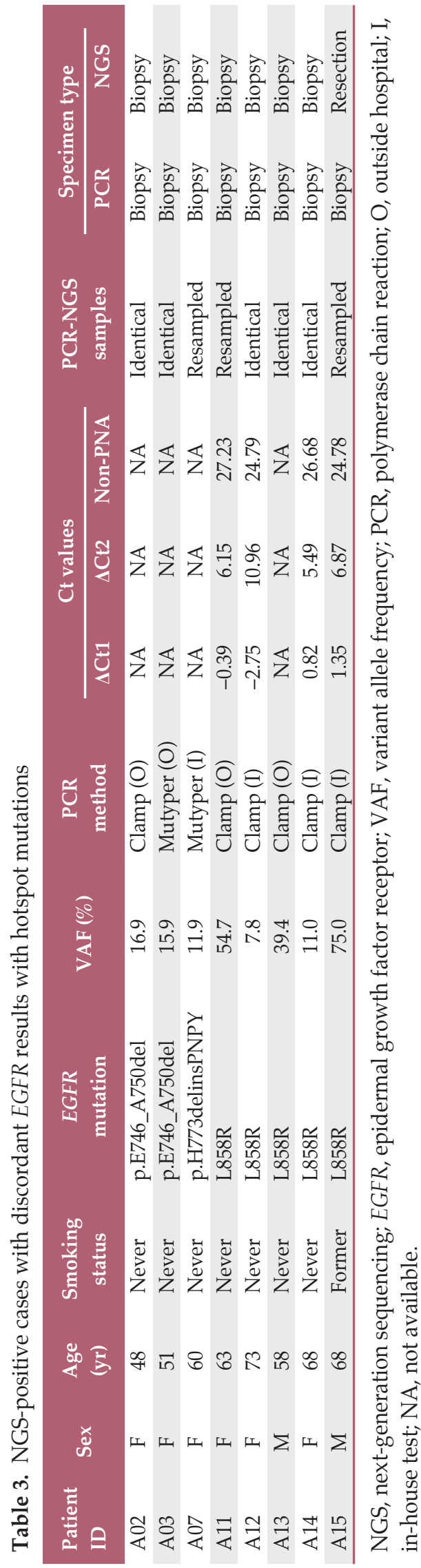

showed responses to therapy (S4 Table). The remaining patients were treated with palliative therapy due to poor general condition, or received cancer immunotherapy, or expired.

\section{Discussion}

According to the NGS results of the present study, the overall probabilities detecting of EGFR, ALK, and ROS1 alterations were $34.0 \%, 3.3 \%$, and $5.8 \%$, respectively. In our cohort, the NGS testing was primarily performed on clinically selected patients who had EGFR/ALK-negative or therapeutic non-responsive or resistant tumors. In contrast, ROS1 IHC/FISH tests were not performed during the initial diagnosis of patients that transferred from other hospitals, and a considerable number of cases were subject to NGS test in our institution without receiving either the ROS1 IHC or ROS1 FISH analyses. Therefore, the NGS-tested cohort was likely enriched in EGFR / ALK-negative cases and could not reflect the general characteristics of lung cancer patients.

The aim of the present study was to compare the results of single-gene assays and NGS testing and to assess the occurrence of false results associated with these methods in the molecular diagnostics of lung cancer. The results presented here indicate that false-negatives occasionally occur in single-gene assays. Regarding NGS results as a final outcome, EGFR PCR showed $80.3 \%$ of sensitivity and $99.4 \%$ of specificity, ALK FISH showed $71.4 \%$ of sensitivity and $100 \%$ of specificity, and ROS1 FISH showed $100 \%$ of sensitivity and 99.5\% of specificity. Positive predictive value of EGFR PCR method, ALK FISH, and ROS1 FISH, was $98.9 \%, 100 \%$, and $67.8 \%$, respectively, and negative predictive value was $88.3 \%$, $98.5 \%$, and $100.0 \%$, respectively (S5 Table). This finding suggests the necessity of re-validating the results of the singlegene assays, especially for negative EGFR assays.

One major reason that the EGFR PCR assay yielded falsenegatives is that the sensitivity of PCR is lower than that of deep targeted NGS. In four cases with EGFR hotspot mutations that were not detected by PCR, all Ct values indicated negative results. However, in two cases of $0<\Delta \mathrm{Ct} 1<2$, a possibility of suboptimal tests was suggested. In these cases, re-test by using twice as high concentration of the sample is recommended. However, re-testing was difficult owing to the small amount of remaining tissue after the pathological and molecular examinations.

It is also notable that a misleading positive result was obtained using the EGFR PCR method. In this case, the positive PCR result was based on the detection of E19del, whereas the positive NGS result was based on the detection of E19 p.L747P. Because the EGFR PCR method used in this study 
Table 4. NGS-positive cases with discordant EGFR results with rare mutations

\begin{tabular}{lcclllllll}
$\begin{array}{l}\text { Patient } \\
\text { ID }\end{array}$ & $\begin{array}{c}\text { Sex } \\
(\mathbf{y r})\end{array}$ & $\begin{array}{c}\text { Age } \\
(\mathbf{y r})\end{array}$ & $\begin{array}{c}\text { Smoking } \\
\text { status }\end{array}$ & \multicolumn{1}{c}{$\begin{array}{c}\text { EGFR } \\
\text { mutations }\end{array}$} & VAF (\%) & $\begin{array}{c}\text { PCR } \\
\text { method }\end{array}$ & $\begin{array}{c}\text { PCR-NGS } \\
\text { samples }\end{array}$ & $\begin{array}{c}\text { Specimen type } \\
\text { PCR }\end{array}$ \\
A01 & F & 73 & Never & p.E709_T710delinsD & 7.0 & Clamp (O) & Resampled & Biopsy & Biopsy \\
A04 & F & 44 & Never & p.A750_I759>PL & 12.6 & Clamp (O) & Identical & Biopsy & Biopsy \\
A05 & F & 41 & Never & p.D770_N771insG & 11.1 & Clamp (O) & Resampled & Biopsy & Biopsy \\
A06 & M & 61 & Former & p.D770_N771insGD & 37.2 & Clamp (I) & Identical & Biopsy & Biopsy \\
A08 & F & 46 & Never & p.A767delinsASVD & 4.2 & Clamp (I) & Identical & Biopsy & Biopsy \\
A09 & M & 64 & Never & p.A767_V769dup & 25.0 & Clamp (I) & Identical & Resection & Resection \\
A10 & F & 62 & Never & p.D770_N771insGF & 81.2 & Clamp (O) & Resampled & Biopsy & Biopsy \\
A16 & F & 67 & Never & p.E709_T710delinsD & 75.0 & Mutyper (I) & Resampled & Biopsy & Resection \\
\hline
\end{tabular}

NGS, next-generation sequencing; EGFR, epidermal growth factor receptor; VAF, variant allele frequency; PCR, polymerase chain reaction; $\mathrm{O}$, outside hospital; I, in-house test.

A
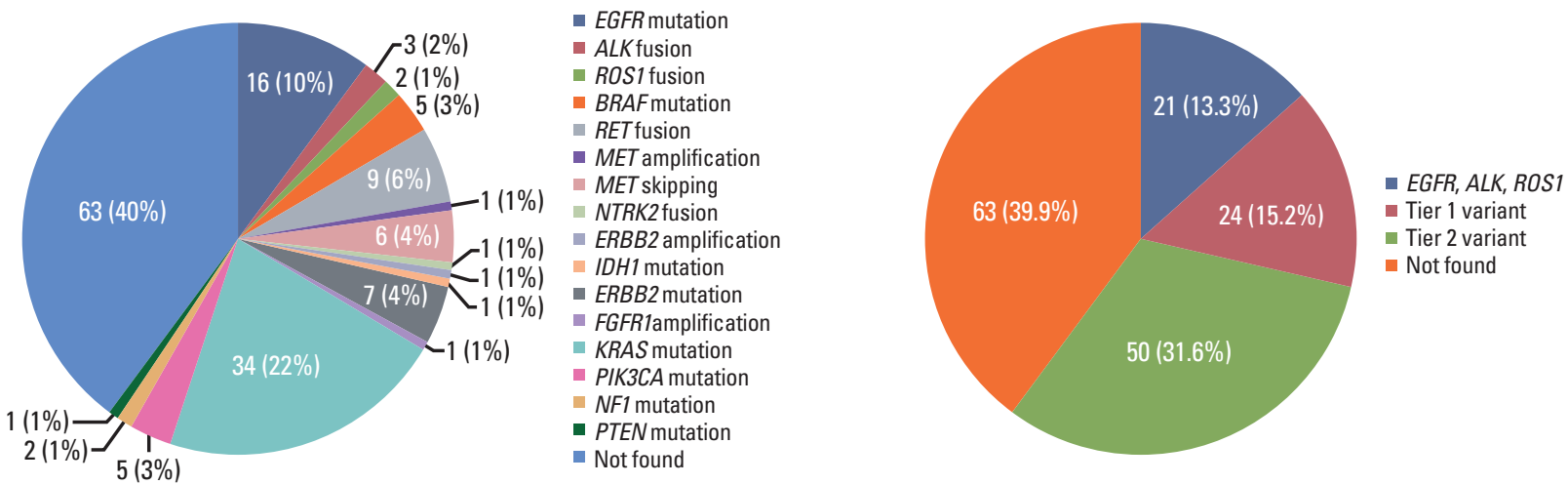

Fig. 1. Next-generation sequencing (NGS)-detected alterations identified in 158 cases previously designated as wild-type. (A) Distribution of potentially actionable genetic alterations. (B) Distribution of genetic alterations. Variant classification was based on recommendations from the Association for Molecular Pathology, American Society of Clinical Oncology, and College of American Pathologists [8]. EGFR, epidermal growth factor receptor; ALK, anaplastic lymphoma kinase; ROS1, ROS proto-oncogene 1; IDH1, isocitrate dehydrogenase 1; FGFR1, fibroblast growth factor receptor 1; PIK3CA, phosphatidylinositol-4, 5-bisphosphate 3-kinase catalytic subunit alpha; NF1, neurofibromin 1.

was designed to only detect deletion-type mutations in exon 19 , the true alteration (i.e., base substitution) was misinterpreted. This is concerning because the EGFR p.L747P mutation had been rarely reported with relation to poor response to gefitinib and erlotinib, in contrast to the favorable therapeutic response of E19del mutation [9-11]. Indeed, this specific case was resistant to gefitinib treatment, thereby confirming the conclusion that accurate mutation identification can impact clinical management.

Another inherent limitation of the PCR method is that only targeted loci can be identified [12]. In this study, 3.3\% of cases with rare-type EGFR mutations were not detected using
PCR. Rare EGFR mutations had been reported to be observed in male smokers with inferior EGFR TKI response $[13,14]$. However, recent studies have reported that rare EGFR mutations can be more effectively targetable with appropriate TKIs [15]. Similarly, several recent studies have reported that insertions in EGFR exon 20, which had been known to be resistant to first- or second-generation EGFR TKI treatments, could be effectively targeted using new TKIs, such as poziotinib [16-18]. Therefore, rare EGFR mutations could be equally important targets in the treatment of advanced-stage lung cancer, and detailed information regarding EGFR mutation type is needed to apply appropriate therapies. Based on our 
finding, NGS testing could be used as a complementary method to overcome the limitations of conventional PCR methods.

We also identified some limitations in the FISH assays, with false-negative $(n=2)$ and false-positive $(n=1)$ results observed for the ALK FISH and ROS1 FISH assays, respectively. For the two ALK FISH-negative cases, the FISH tests had been performed at other hospitals. In both cases, the $A L K$ IHC results indicated moderate protein expression, whereas the FISH results indicated low rates of split signals, which implies that the false-negatives could have resulted from the technical or interpretational aspects of FISH [19-21]. Notably, both patients were eventually treated with crizotinib and alectinib, respectively, and exhibited clinical responses. Meanwhile, for the ROS1 FISH false-positive case, the fusion identified by the FISH assay was determined to be a rare intronic fusion, which does not generate an active functional protein change, and the error was further empowered by non-specific positive ROS1 IHC results.

Several other studies have also reported the possibility of false results from FISH assays and have strongly suggested that NGS be used to resolve IHC-FISH discordant cases [20-24]. In addition, the re-validation of molecular testing would be useful in the treatment of tumors that are unresponsive to target agents. Currently, both ALK IHC and ROS1 real-time PCR kits have been approved for use as companion diagnostics in Korea and are expected to increase the diagnostic yield of patients harboring gene fusions. However, when compared to NGS methods, these tests are limited in their ability to accurately identify fusion partners or fusion variants that additional verification in clinical practice may provide further insight.

In the present study, NGS testing identified EGFR / ALK/ ROS1 alterations in $13.3 \%$ of previously diagnosed as wildtype cases using single-gene assay. These additional cases were mostly females or never smokers, although males and smokers were also included. In addition, other targetable alterations were identified in $36.1 \%$ of the "wild-type" cases. These results might suggest the implementation of NGS as a first-line test for molecular diagnostics of lung cancer. However, at the present time, it is still challenging to use NGS as first method in Korea because of high costs and the necessity

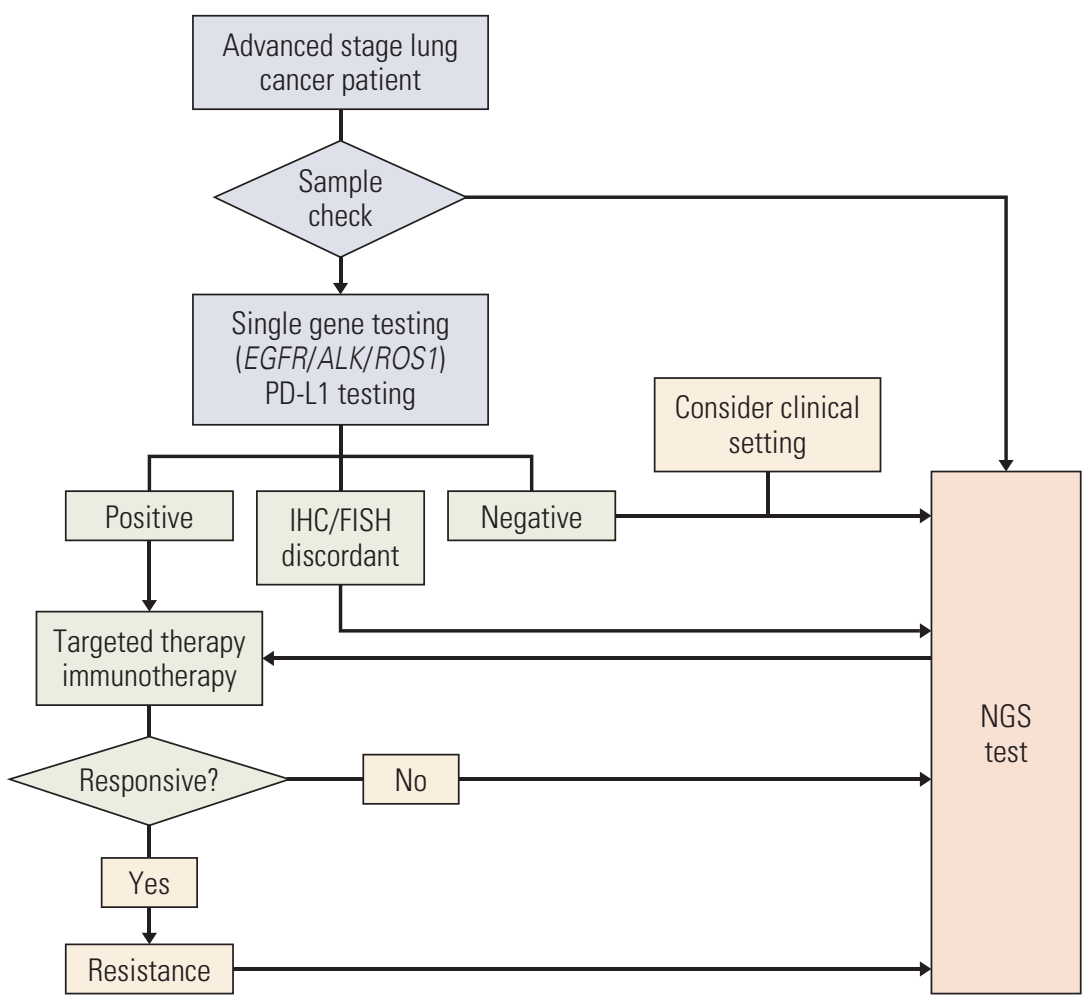

Fig. 2. Proposed algorithm for the molecular diagnostics of advanced-stage lung cancer patients. In terms of turnaround time and frequency of major genetic alterations, single-gene testing can be performed first. When results of single-gene assays are negative or clinically indicated, next-generation sequencing (NGS) testing should be performed. The NGS testing can be performed first, so this is also shown in this figure. EGFR, epidermal growth factor receptor; ALK, anaplastic lymphoma kinase; ROS1, ROS proto-oncogene 1; IHC, immunohistochemistry; FISH, fluorescence in situ hybridization. 
of specialized implements and bioinformatics of NGS. In addition, current resources for NGS analysis result in much longer turnaround time compared to conventional tests. Regarding well-established single-gene assays in Korea and high prevalence of EGFR mutations in Korean lung cancer patients [25], single-gene tests are still indispensable. Thus, we recommend maintaining single-gene tests as first-line tests and actively use NGS for re-validation of the negative results. Oncologists and pathologists should consider the limitations of conventional methods and re-validate the results of these methods, especially when targetable alterations are not detected in females or never smokers, targets are detected but no therapeutic response is observed, or negative results could be the product of insufficient sample quantity (Fig. 2). Notably, in our cohort, of patients with additionally identified actionable alterations, $32.6 \%$ received matched therapy with a clinical benefit of $48.4 \%$. These results are similar to those described in the report by Jordan et al. [3].

There are some limitations to our study. First, this study was limited by its retrospective design. Second, as previously mentioned, our cohort does not reflect the general characteristics of advanced-stage lung cancer patients, since the NGStested cohort was likely enriched in EGFR/ALK-negative cases and since a high proportion of the patients were female or never smokers.

In conclusion, even though the conventional and NGS methods were concordant in the majority of cases, NGS testing still revealed a considerable number of additional EGFR, $A L K$, and ROS1 alterations, as well as other targetable alterations, in Korean advanced-stage lung cancer patients. Given the high frequency of EGFR and other targetable mutations identified in the present study, NGS testing is highly recommended in the diagnosis of Korean lung cancer patients.

\section{Electronic Supplementary Material}

Supplementary materials are available at Cancer Research and Treatment website (https:// www.e-crt.org).

\section{Conflicts of Interest}

Conflict of interest relevant to this article was not reported.

\section{Acknowledgments}

The present study was supported by a Basic Science Research Program grant (NRF-2018R1D1A1B07047811) through the National Research Foundation of Korea (NRF), which is funded by the Ministry of Education, Korea.

\section{References}

1. Lindeman NI, Cagle PT, Aisner DL, Arcila ME, Beasley MB, Bernicker $\mathrm{EH}$, et al. Updated molecular testing guideline for the selection of lung cancer patients for treatment with targeted tyrosine kinase inhibitors: guideline from the College of American Pathologists, the International Association for the Study of Lung Cancer, and the Association for Molecular Pathology. J Thorac Oncol. 2018;13:323-58.

2. Shim HS, Choi YL, Kim L, Chang S, Kim WS, Roh MS, et al. Molecular testing of lung cancers. J Pathol Transl Med. 2017; 51:242-54.

3. Jordan EJ, Kim HR, Arcila ME, Barron D, Chakravarty D, Gao J, et al. Prospective comprehensive molecular characterization of lung adenocarcinomas for efficient patient matching to approved and emerging therapies. Cancer Discov. 2017;7:596609.

4. Hagemann IS, Devarakonda S, Lockwood CM, Spencer DH, Guebert K, Bredemeyer AJ, et al. Clinical next-generation sequencing in patients with non-small cell lung cancer. Cancer. 2015;121:631-9.

5. Pennell NA, Mutebi A, Zhou ZY, Ricculli ML, Tang W, Wang $\mathrm{H}$, et al. Economic impact of next generation sequencing vs sequential single-gene testing modalities to detect genomic alterations in metastatic non-small cell lung cancer using a decision analytic model. J Clin Oncol. 2018;36(15 Suppl):9031.

6. Shao D, Lin Y, Liu J, Wan L, Liu Z, Cheng S, et al. A targeted next-generation sequencing method for identifying clinically relevant mutation profiles in lung adenocarcinoma. Sci Rep. 2016;6:22338.

7. Cha YJ, Lee JS, Kim HR, Lim SM, Cho BC, Lee CY, et al. Screening of ROS1 rearrangements in lung adenocarcinoma by immunohistochemistry and comparison with ALK rearrangements. PLoS One. 2014;9:e103333.

8. Li MM, Datto M, Duncavage EJ, Kulkarni S, Lindeman NI, Roy $S$, et al. Standards and guidelines for the interpretation and reporting of sequence variants in cancer: a joint consensus recommendation of the Association for Molecular Pathology, American Society of Clinical Oncology, and College of American Pathologists. J Mol Diagn. 2017;19:4-23.

9. Legras A, Barritault M, Tallet A, Fabre E, Guyard A, Rance B, et al. Validity of targeted next-generation sequencing in routine care for identifying clinically relevant molecular profiles in non-small-cell lung cancer: results of a 2-year experience on 1343 samples. J Mol Diagn. 2018;20:550-64.

10. Yu G, Xie X, Sun D, Geng J, Fu F, Zhang L, et al. EGFR muta- 
tion L747P led to gefitinib resistance and accelerated liver metastases in a Chinese patient with lung adenocarcinoma. Int J Clin Exp Pathol. 2015;8:8603-6.

11. Wu SG, Gow CH, Yu CJ, Chang YL, Yang CH, Hsu YC, et al. Frequent epidermal growth factor receptor gene mutations in malignant pleural effusion of lung adenocarcinoma. Eur Respir J. 2008;32:924-30.

12. Tuononen K, Maki-Nevala S, Sarhadi VK, Wirtanen A, Ronty $\mathrm{M}$, Salmenkivi $\mathrm{K}$, et al. Comparison of targeted next-generation sequencing (NGS) and real-time PCR in the detection of EGFR, KRAS, and BRAF mutations on formalin-fixed, paraffin-embedded tumor material of non-small cell lung carcinoma-superiority of NGS. Genes Chromosomes Cancer. 2013; 52:503-11.

13. Kauffmann-Guerrero D, Huber RM, Reu S, Tufman A, Mertsch $\mathrm{P}$, Syunyaeva Z, et al. NSCLC patients harbouring rare or complex EGFR mutations are more often smokers and might not benefit from first-line tyrosine kinase inhibitor therapy. Respiration. 2018;95:169-76.

14. Tu HY, Ke EE, Yang JJ, Sun YL, Yan HH, Zheng MY, et al. A comprehensive review of uncommon EGFR mutations in patients with non-small cell lung cancer. Lung Cancer. 2017; 114:96-102.

15. Kobayashi Y, Mitsudomi T. Not all epidermal growth factor receptor mutations in lung cancer are created equal: Perspectives for individualized treatment strategy. Cancer Sci. 2016; 107:1179-86.

16. Vyse S, Huang PH. Targeting EGFR exon 20 insertion mutations in non-small cell lung cancer. Signal Transduct Target Ther. 2019;4:5

17. Voon PJ, Tsui DW, Rosenfeld N, Chin TM. EGFR exon 20 insertion A763-Y764insFQEA and response to erlotinib: letter. Mol Cancer Ther. 2013;12:2614-5.

18. Robichaux JP, Elamin YY, Tan Z, Carter BW, Zhang S, Liu S, et al. Mechanisms and clinical activity of an EGFR and HER2 exon 20-selective kinase inhibitor in non-small cell lung cancer. Nat Med. 2018;24:638-46.

19. Rosenbaum JN, Bloom R, Forys JT, Hiken J, Armstrong JR, Branson J, et al. Genomic heterogeneity of ALK fusion breakpoints in non-small-cell lung cancer. Mod Pathol. 2018;31:791808.

20. Ali SM, Hensing T, Schrock AB, Allen J, Sanford E, Gowen K, et al. Comprehensive genomic profiling identifies a subset of crizotinib-responsive ALK-rearranged non-small cell lung cancer not detected by fluorescence in situ hybridization. Oncologist. 2016;21:762-70.

21. Pekar-Zlotin M, Hirsch FR, Soussan-Gutman L, Ilouze M, Dvir A, Boyle T, et al. Fluorescence in situ hybridization, immunohistochemistry, and next-generation sequencing for detection of EML4-ALK rearrangement in lung cancer. Oncologist. 2015;20:316-22.

22. van der Wekken AJ, Pelgrim R, 't Hart N, Werner N, Mastik MF, Hendriks L, et al. Dichotomous ALK-IHC is a better predictor for ALK inhibition outcome than traditional ALK-FISH in advanced non-small cell lung cancer. Clin Cancer Res. 2017;23:4251-8.

23. Cabillic F, Hofman P, Ilie M, Peled N, Hochmair M, Dietel M, et al. ALK IHC and FISH discordant results in patients with NSCLC and treatment response: for discussion of the question-to treat or not to treat? ESMO Open. 2018;3:e000419.

24. Lin C, Shi X, Yang S, Zhao J, He Q, Jin Y, et al. Comparison of ALK detection by FISH, IHC and NGS to predict benefit from crizotinib in advanced non-small-cell lung cancer. Lung Cancer. 2019;131:62-8.

25. Choi CM, Kim HC, Jung CY, Cho DG, Jeon JH, Lee JE, et al. Report of the Korean Association of Lung Cancer Registry (KALC-R), 2014. Cancer Res Treat. 2019;51:1400-10. 